\title{
Trapped radioactive isotopes for fundamental symmetry investigations
}

\section{The TRI $\mu$ P Facility}

\author{
Lorenz Willmann • Klaus Jungmann • Cornelis J. G. Onderwater • \\ Rob G. E. Timmermans • Hans W. Wilschut
}

Published online: 3 April 2012

(C) The Author(s) 2012. This article is published with open access at Springerlink.com

\begin{abstract}
Discrete symmetries tested in high precision atomic physics experiments provide guidance to model building beyond the Standard Model (SM). Here experimental opportunities arise for searches for permanent electric dipole moments (EDMs) and measurements of atomic parity violation (APV). Heavy atoms are favorable for such experiments since symmetry violating effects in atoms increase faster than the third power of the nuclear charge $\mathrm{Z}$. Of special interest are isotopes of the heavy alkaline earth element radium $(Z=88)$ since they offer large enhancement factors for EDMs and provide a new experimental road towards high precision measurements of atomic parity violation. These opportunities are exploited at the TRI $\mu$ P facility at KVI, Groningen.
\end{abstract}

Keywords Fundamental symmetries • Permanent electric dipole moments • Atomic parity violation $\cdot$ Radioactive beam facilities

\section{Introduction}

The Standard Model (SM) comprises the knowledge of physics over a wide range of energies and different interactions. Its success is visible through predictions which were verified by experimental observations. All experimental observed phenomena are accommodated by the theory, but the SM does not provide explanations for them, e.g. the origin of parity violation or the matter-antimatter asymmetry in the universe [1]. Here, experimental limits on violations of discrete symmetries, i.e. parity (P), particle-antiparticle symmetry (charge conjugation, $\mathrm{C}$ ) and time reversal (T) provide sensitive tests of the range of validity of the SM which guide theoretical model building beyond the SM.

L. Willmann $(\bowtie) \cdot$ K. Jungmann · C. J. G. Onderwater · R. G. E. Timmermans · H. W. Wilschut KVI, University of Groningen, Groningen, Netherlands e-mail: willmann@kvi.nl 
The heavy alkaline earth element radium is at the focus of this paper. This system is preferred since symmetry violating effects scale with higher powers of the nuclear charge $\mathrm{Z}$ due to the larger number of coherently contributing particles and relativistic effects $[2,3]$. In addition, some isotope specific enhancement factors are particularly large for neutral radium isotopes. In the case of permanent electric dipole moments (EDMs) which violate $\mathrm{P}$ and $\mathrm{T}$ at the same time, these can be as large as $4-5$ orders of magnitude. The nuclear [4] and unique atomic structure [5, 6] of radium lead to this enhancements compared to ${ }^{199} \mathrm{Hg}$ in which the strongest experimental limit for a nuclear EDM of $3.1 \cdot 10^{-29} \mathrm{ecm}$ has been determined [7]. Further interest in radium isotopes arises from the possible quantitative measurement of atomic parity violation (APV) in a single trapped ion [8] and in particular for radium isotopes $[9,10]$. An experiment on a single trapped $\mathrm{Ra}^{+}$ion at KVI aims at a 5-fold improved determination of the weak interaction in atoms compared the previous best measurement Cs [11].

At the TRI $\mu$ P Facility, KVI, Groningen, The Netherlands, a wide range of short lived isotopes are available by online production [12]. The range of ${ }^{209-214} \mathrm{Ra}$ isotopes is produced with ${ }^{204,206} \mathrm{~Pb}$ beams using the AGOR cyclotron. The primary beam power of up to $200 \mathrm{~W}$ impinges on a rotating target with pyrolytic graphite foils of $2 \mathrm{mg} / \mathrm{cm}^{2}$ thickness. After the magnetic separator the Ra isotopes are stopped and re-ionized to $\mathrm{Ra}^{+}$in a Thermal Ionizer (TI) [13]. They are separated from other ions in a Wien Filter and cooled in a gas-filled Radio Frequency Quadrupole (RFQ). The rate of $\mathrm{Ra}^{+}$after the TI is up to $5 \times 10^{4} / \mathrm{s}$ depending on the isotope. These isotopes are exploited in our searches for permanent electric dipole moments and measurements of atomic parity violation.

\section{Electric dipole moments}

A wide range of different experimental searches for EDMs from elementary particles up to complex systems like molecules are currently underway [14, 15]. EDMs exhibit a robust discovery potential for physics beyond the SM since their values within the SM are well below current experimental sensitivities. However, their sizes in a number of proposed extensions of the SM attempting to explain features like parity violation or baryon number asymmetry appear in the range of ongoing experimental efforts. Electron and nuclear EDMs are strongly enhanced in radium isotopes. The nuclear octupole deformations of isotopes near the valley of stability result in an enhancement of 2-3 orders of magnitude for nuclear EDMs [4]. In addition, the unique atomic structure of Ra gives rise to enhancements of 4-5 orders of magnitude for a nuclear EDM in the metastable $7 \mathrm{~s} 6 \mathrm{~d}{ }^{3} \mathrm{D}_{2}$ state and a factor of 5000 for the electron EDM in the $7 \mathrm{~s} 6 \mathrm{~d}{ }^{3} \mathrm{D}_{1}$ state [5]. Radium EDM experiments with an anticipated level of sensitivity of $10^{-30} \mathrm{ecm}$ are currently prepared at the Argonne National Laboratory (ANL), Il, USA on the ground state of ${ }^{225} \mathrm{Ra}[16]$ and at KVI where the focus is on the exploitation of the large atomic enhancement factors in the metastable D-states for electron and nuclear EDMs.

A crucial step toward the EDM searches is the efficient capture of radium in atomic traps. At KVI we have developed the laser cooling and trapping of heavy alkaline earth elements on the strong singlet-singlet ${ }^{1} \mathrm{~S}_{0}-{ }^{1} \mathrm{P}_{1}$ transition with barium [17], the chemical homologue of radium. The efficient laser cooling scheme involves 


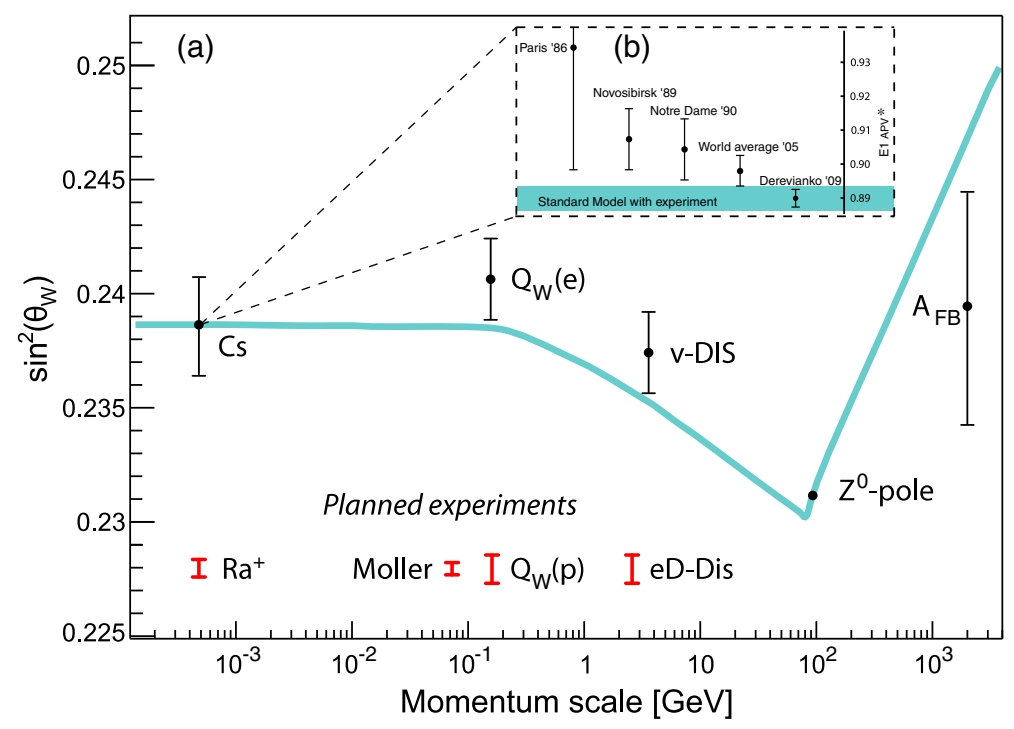

Fig. 1 (a) The weak mixing angle $\Theta_{W}$ in the Standard Model shows an energy scale dependent behavior due to radiative corrections. There are experiments planned at different energy scales to measure this dependence. Atomic parity violation provides a measurement at the lowest energy scale. Up to now a measurement in Cs $[11,20]$ provides the best results. APV measurements in a single trapped $\mathrm{Ra}^{+}$have the potential for a 5-fold improvement over the Cs results. (b) The inset shows the development of accuracy and precision of theoretical calculations of APV in Cs [20] over time. The anticipated precision of planned experiments is also given

seven laser at a time. The lifetime of the trapped barium atoms is about $1.5 \mathrm{~s}$. The capture efficiency of barium atoms from a thermal beam into the magneto optical trap was determined to $0.5 \%$ which was limited by laser power for transitions from the metastable D-states significantly. Another approach for laser cooling and trapping of radium has been pursued by the group at ANL. However, the weak ${ }^{1} \mathrm{~S}_{0}$ ${ }^{3} \mathrm{P}_{1}$ intercombination line provides a 150 times smaller optical force than the strong ${ }^{1} \mathrm{~S}_{0}-{ }^{1} \mathrm{P}_{1}$ transition resulting in a capture efficiency of $10^{-6}$ [18]. The application of the laser cooling method developed at KVI with Ba promises thus a significant gain in sensitivity for Ra EDM searches due to the selection of a new sensitive system and a new type of experimental approach. The combination of the availability of the radium isotopes at online facilities and experimental techniques like laser cooling and optical dipole trapping for radium [19] opens the path to sensitive EDM searches.

\section{Atomic parity violation in a single trapped radium ion}

A single radium ion in an ion trap opens the path to a high precision measurement of atomic parity violation (APV). The experiment at KVI aims at a determination of the nuclear spin independent part of the electroweak interaction at low energy scales in order to extract the weak mixing (Weinberg) angle $\Theta_{W}$. Such a measurement can be compared to other determinations of $\Theta_{W}$ at different energy scales and to the predictions of the SM (Fig. 1). The best measurement of weak interactions in atomic 
Fig. 2 Hyperfine structure of the $6 d^{2} D_{3 / 2}-7 p^{2} P_{3 / 2}$ transition in ${ }^{213} \mathrm{Ra}$ at a wavelength of $1079 \mathrm{~nm}$. The signal is obtained with a few hundred ions trapped and buffer gas cooled ions in a linear Paul trap [9]

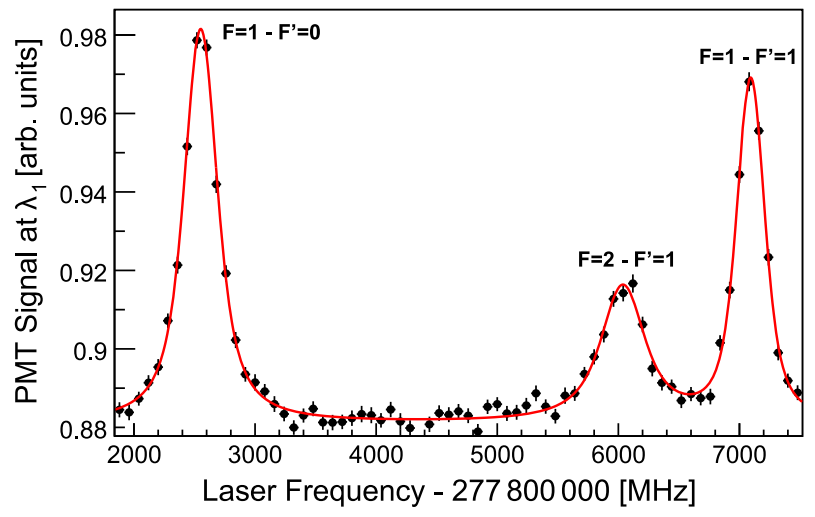

systems has been performed with Cs [11]. The exploitation of the precision towards the weak mixing angle required an extensive treatment of the atomic theory [20] over the years. A single trapped radium ion aims at a 5-fold improvement over the Cs result. This becomes possible due to a new signature of APV due to differential light shifts [8] and the large weak charge due to the large $\mathrm{Z}$ of radium [10].

Spectroscopic information on singly charged radium was limited until recently. However, the exploitation of the sensitivity of a radium ion relies on high precision atomic structure calculations which require experimental input to evaluate their accuracy. We have determined the hyperfine structure in the $6 d^{2} \mathrm{D}_{3 / 2}$ state for ${ }^{209,211,213} \mathrm{Ra}$, the lifetime of the $6 d^{2} \mathrm{D}_{3 / 2}$ state [9, 21] and isotopes shifts [22] for the ${ }^{209-214} \mathrm{Ra}$ isotopes. The hyperfine structure of ${ }^{213} \mathrm{Ra}$ obtained with buffer gas cooled ions in a linear Paul-trap is given in Fig. 2. A comparison of the laser spectroscopy results with the atomic structure calculations permits an estimate on the current accuracy of the theoretical description. The hyperfine structure splittings, which are a sensitive probe of the atomic wave functions in the nuclear region, are in agreement with theory on the level of a few \% [9]. This tests the predictions of APV matrix elements to about the same accuracy [10,23]. Several groups are working on the improvement of the calculations [24]. The trapping of a single radium ion and its laser cooling to the motional ground state of the trap is currently underway.

Progress in the field of experimental investigations of fundamental symmetries benefits from new systems and experimental approaches. The single trapped radium ion can also be exploited as an optical clock [25] which underlines the fruitful crossfertilization between different areas in physics. The Ra ion clock exhibits different sensitivity to temporal changes of the fine structure constant $\alpha$ than other available atomic clocks [25] which makes it an ideal candidate for measurements of time variation of fundamental constants.

Open Access This article is distributed under the terms of the Creative Commons Attribution License which permits any use, distribution, and reproduction in any medium, provided the original author(s) and the source are credited. 


\section{References}

1. Sakharov, A.D.: Pisma Zh. Eksp. Teor. Fiz. 5, 32-35 (1967); translation in JETP Lett. 5, 24-27 (1967)

2. Sandars, P.G.H.: Phys. Lett. 14, 194 (1965)

3. Bouchiat, M.A., Bouchiat, C.C.: Phys. Lett. B 48, 111 (1974)

4. Engel, J., Bender, M., Dobaczewski, J., Jesus, J. Hd., Olbratowski, P.: Phys. Rev. C 68, 025501 (2003); Dobaczewski, J., Engel, J.: Phys. Rev. Lett. 94, 232502 (2005)

5. Flambaum, V.V.: Phys. Rev. A 60, R2611 (1999)

6. Dzuba, V.A., Flambaum, V.V., Ginges, J.S.M.: Phys. Rev. A 61, 062509 (2000)

7. Griffith, W.C., Swallows, M.D., Loftus, T.H., Romalis, M.V., Heckel, B.R., Fortson, E.N.: Phys. Rev. Lett. 102, 101601 (2009)

8. Fortson, N.: Phys. Rev. Lett. 70, 2383 (1993)

9. Versolato, O.O., Giri, G.S., Wansbeek, L.W., Kruithof, W., van der Hoek, D.J., Santra, B., Shidling, P., Onderwater, C.J.G., Willmann, L., Wilschut, H.W., Timmermans, R.G.E., Jungmann, K.: Phys. Rev. A 82, 010501(R) (2010)

10. Wansbeek, L.W., Sahoo, B.K., Timmermans, R.G.E., Jungmann, K., Das, B.P., Mukherjee, D.: Phys. Rev. A 78, 050501(R) (2008)

11. Bennett, S.C., Wieman, C.E.: Phys. Rev. Lett. 82, 2484 (1999)

12. Berg, G.P.A., Dermois, O.C., Dammalapati, U., et al.: Nucl. Inst. Meth. A 560, 169 (2006)

13. Shidling, P.D., Giri, G.S., van der Hoek, D.J., et al.: Nucl. Instr. Meth. A 622, 11 (2010)

14. Commins, E.D.: J. Phys. Soc. Jpn. 76, 111010 (2007)

15. Onderwater, C.J.G.: this volume

16. Holt, R.J., Ahmad, I., Bailey, K., Graner, B., Greene, J.P., Korsch, W., Lu, Z.-T., Mueller, P., O’Conner, T.P., Sulai, I.A., Trimble, W.L.: Nucl. Phys. A 844, 53 (2010)

17. De, S., Dammalapati, U., Jungmann, K., Willmann, L.: Phys. Rev. A 79, 041402R (2009)

18. Guest, J.R., Scielzo, N.D., Ahmad, I., Bailey, K., Greene, J.P., Holt, R.J., Lu, Z.-T., O'Connor, T.P., Potterveld, D.H.: Phys. Rev. Lett. 98, 093001 (2007)

19. Dammalapati, U., Santra, B., Willmann, L.: J. Phys. B: At. Mol. Opt. Phys. 45, 025001 (2012)

20. Porsev, S.G., Beloy, K., Derevianko, A.: Phys. Rev. Lett. 102, 181601 (2009)

21. Versolato, O.O., Giri, G.S., van den Berg, J.E., Boll, O., Dammalapati, U., van der Hoek, D.J., Hoekstra, S., Jungmann, K., Kruithof, W.L., Muller, S., Nuñez Portela, M., Onderwater, C.J.G., Santra, B., Timmermans, R.G.E., Wansbeek, L.W., Willmann, L., Wilschut, H.W.: Phys. Lett. A 375, 3130 (2011)

22. Giri, G.S., Versolato, O.O., vd Berg, J.E., Dammalapati, U., vd Hoek, D.J., Jungmann, K., Kruithof, W., Nuñez Portela, M., Onderwater, C.J.G., Santra, B., Timmermans, R.G.E., Wansbeek, L.W., Willmann, L., Wilschut, H.W.: Phys. Rev. A 84, 020503R (2011)

23. Sahoo, B.K., Das, B.P., Chaudhuri, R.K., Mukerjee, D., Timmermans, R.G.E., Jungmann, K.: Phys. Rev. A 76, 040504 (2007)

24. Dzuba, V.A., Flambaum, V.V.: arXiv:1104.0086 [physics.atom-ph]; Pal, R., Jiang, D., Safonova, M.S., Safronova, U.I.: Phys. Rev. A 79, 062505 (2009); Sahoo, B.K., Mandal, P., Mukkerjee, M.: Phys. Rev. A 83, 030502(R) (2011)

25. Versolato, O.O., Wansbeek, L.W., Jungmann, K., Timmermans, R.G.E., Willmann, L., Wilschut, H.W.: Phys. Rev. A 83, 043829 (2011) 